\title{
FWM-based, Idler-free Phase Quantiser with Flexible Operating Power
}

\author{
K. R. H. Bottrill*, L. Jones, F. Parmigiani, D. J. Richardson, P. Petropoulos \\ Optoelectronics Research Centre, University of Southampton, SO17 1BJ, UK. \\ *krhb1g12@soton.ac.uk
}

\begin{abstract}
Coherently adding a signal's conjugate and third harmonic at the latter's wavelength enables phase quantisation across a large operating power range. With broadband phase noise, a 5.6 dB QPSK receiver sensitivity improvement is achieved with $\mathrm{BER}=10^{-4}$.
\end{abstract}

OCIS codes: $060.1155,060.4370,060.0060,060.5060,060.2360$.

\section{Introduction}

Given that two major benefits often cited in favour of all-optical signal processing over its electronic equivalent are decreased energy consumption and reduced system complexity, it is pertinent to seek out all-optical phase regenerating schemes which exhibit these particular qualities. For the specific case of 4-level phase quantisation (as applicable to QPSK - Quaternary Phase Shift Keying signals), phase sensitive amplifier (PSA) based schemes, often involve the coherent addition of conjugated $3^{\text {rd }}$ harmonic photons (and generally, for $M$-level quantisation, conjugated $(M-$ $1)^{\text {th }}$ harmonic photons) to the original signal $[1,2]$ or alternatively the decomposition of the QPSK signal into two BPSK signals which are then regenerated separately, before being coherently recombined $[3,4]$. We propose and demonstrate a wavelength-converting, idler-free, phase quantising scheme which, as opposed to coherently adding harmonic photons to the signal channel, coherently adds conjugated signal photons to the harmonic channel. The use of separate wavelength to the original signal allows the capability to independently control their relative power weights, achieving phase squeezing over a variety of operating powers, potentially making it a particularly low power solution for phase regeneration whilst offering a compact, single nonlinear stage setup. We implement this system and analyse its regenerative performance in terms of its bit error ratio (BER) curves and constellation plots for a broadband phase noise loaded QPSK signal.

\section{Concept}

Many PSA based schemes for 4-level phase quantisation make use of two optical nonlinear processing stages [1, 2]; the first consists of a degenerate, single pump FWM scheme to produce high-order harmonics, phase locked to the signal, of which the $3^{\text {rd }}$ is chosen to be mixed back into the signal through a second stage of FWM, as illustrated in Fig. 1-a).

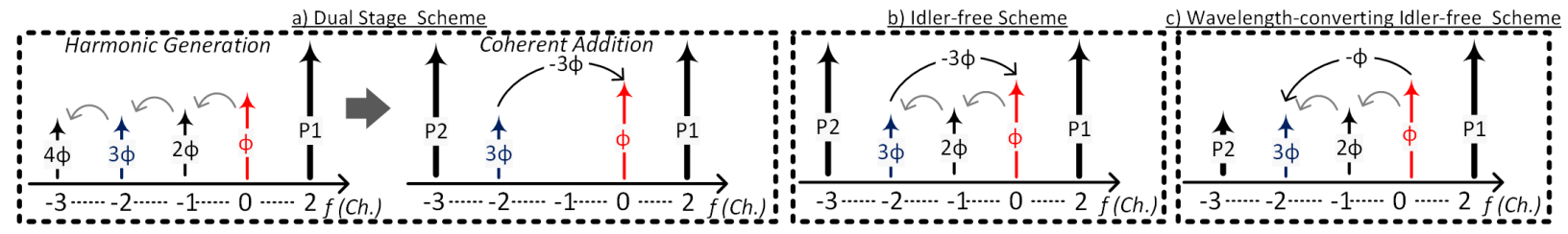

Fig. 1: Illustration of three spectral configurations for achieving PSA. Curved arrows represent individual FWM interactions.

A variant of this scheme (shown in Fig. 1-b)), referred to as the idler-free scheme [5], effectively combines these two stages into a single one by making use of two pumps with their power tuned so as to simultaneously produce the $3^{\text {rd }}$ phase harmonic and mix it back into the signal. Although this scheme is compact, using only a single stage, high pump powers must be used to produce enough harmonic and coherently add it back onto the signal, with the scheme only operating for very specific pump-signal power ratios.

The proposed wavelength-converting, idler-free scheme is illustrated in Fig. 1-c). The setup is similar to the idlerfree scheme shown in Fig. 1 b), but with the two pumps asymmetric in power. Instead of producing the $3^{\text {rd }}$ harmonic and coherently adding its conjugate to the original signal, phase conjugated photons of the signal are coherently added to the $3^{\text {rd }}$ phase harmonic. As a consequence, the regenerated signal is formed at the wavelength of the $3^{\text {rd }}$ phase 
harmonic and is phase conjugated. Although the two nonlinear processes (harmonic generation and coherent addition) occur simultaneously, their contributions to the output may now be controlled independently. Hence, for any specific signal and P1 power values it is possible to achieve, provided sufficient OSNR (optical signal to noise ratio), any level of phase squeezing, simply by properly adjusting the power of P2, potentially allowing lower pump powers to be used compared to the standard idler-free scheme. The system can either be followed by a wavelength converter to obtain output at the original signal wavelength or indeed used as a phase regenerating wavelength converter in its own right.

\section{Experimental Setup}
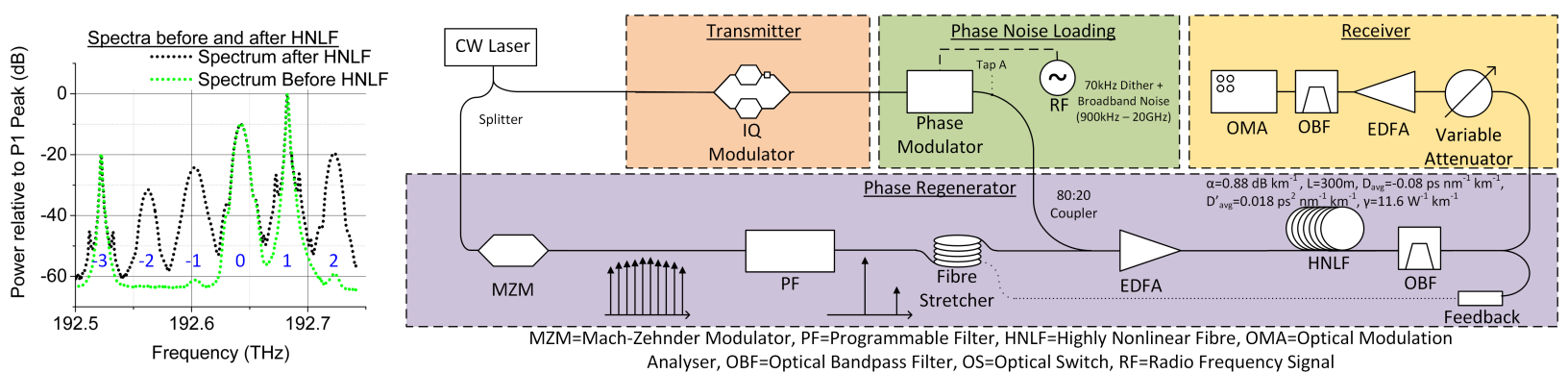

Fig. 2: Left: Spectra before and after HNLF. Right: Wavelength-converting idler-free phase quantiser setup.

A schematic of the experimental setup is given in Fig. 2. A $192.5 \mathrm{THz}(1557.36 \mathrm{~nm})$ laser source is split into two paths, with one part being passed to the transmitter to produce a $10 \mathrm{Gbaud}$ non-return-to-zero QPSK signal carrying two quadrature multiplexed PRBS15 datastreams and the other part being passed to the phase regenerator. After modulation, the signal is then passed through a phase modulator to have broadband phase noise applied to it, as well as a $70 \mathrm{kHz}$ sinusoidal dither, used for carrier phase tracking (discussed later).

In the phase regeneration section, the laser is passed through a sinusoidally driven Mach-Zehnder modulator (MZM) to produce an optical frequency comb with lines at $192.5+0.04 n \mathrm{THz}$ where $n$ is the channel number, and is consistent with the labelling in Fig. 1. The programmable filter is used to select out the required pump lines and control their power; as per Fig. 1-c), we select Channel 1 (at $192.54 \mathrm{THz}$ ) to be P1 and Channel -3 (at 192.38 THz) to be P2.

The pumps are then combined with the signal using an optical coupler and amplified using an EDFA before being launched into the highly nonlinear fibre (HNLF). Fig. 2 provides spectra before (dotted green line) and after (dotted black line) the HNLF. An optical bandpass filter is then used to select out the regenerated signal, which is located at Channel -2 with centre frequency $192.42 \mathrm{THz}$. A coupler is used to separate a small portion of the signal out and the dither signal extracted electrically to operate the carrier tracking feedback loop, driving a fibre stretcher through which the pumps travelled before they were combined with the signal.

The receiver consists of a variable attenuator followed by an EDFA with fixed power output (to enable control of the signal OSNR), and an optical bandpass filter. Finally, the signal is coherently detected using an optical modulation analyser.

\section{Results}

As the characteristics of the system are highly dependent upon the relative powers of the pumps and the signal, the system's phase transfer and phase dependent gain profiles were measured for a range of pump and signal power combinations by operating the system on a CW signal, following the method described in [6]. Fig. 3-a) shows an example of the phase transfer and phase dependent gain profiles of the system for a total HNLF input power of $24 \mathrm{dBm}$, with the power of pump $\mathrm{P} 1=22 \mathrm{dBm}, \mathrm{P} 2=2 \mathrm{dBm}$ and signal power equal to $20 \mathrm{dBm}$, resulting in a wavelength converted signal OSNR $=35 \mathrm{~dB}$.

Using these power values, the system was assessed through constellation plots (given in Fig. 3-b)) and BER curves (Fig. 3-c)) for a range of phase noise levels. Without any additional noise, the constellation plots show that the regenerated signal (Fig. 3-b), plot 2)) is slightly degraded compared to the unregenerated signal (Fig. 3-b), plot 1)). This is confirmed by the BER curves for which the regenerated signal (open black triangles) suffers a power penalty of $0.5 \mathrm{~dB}$ for a BER of $10^{-4}$, as compared to the unregenerated signal (open black circles); for a BER of $10^{-3}$, the system is operated without penalty.

With a signal phase error of $8^{\circ}$ root mean squared (rms), the constellation plots (Fig. 3-b), plots 3) and 4)) show a noticeable improvement from the use of the regenerator, with a reduction in phase error from $8^{\circ} \mathrm{rms}$ down to $4.5^{\circ} \mathrm{rms}$. 
The corresponding BER curves have been omitted for clarity, but show an improvement in receiver sensitivity of $1 \mathrm{~dB}$ using the regenerator.

The final pair of constellation plots shown (Fig. 3-b), plots 5) and 6)), are those corresponding to a pre-regeneration signal phase error of $12^{\circ} \mathrm{rms}$. Here, the phase error is quite substantial, but with regeneration, it is reduced to $5^{\circ} \mathrm{rms}$. The corresponding BER plots for this input noise level are shown in green in Fig. 3-c), with the input signal plotted with open circles, and the regenerated signal plotted with open triangles. Here, there is a dramatic improvement from the use of the regenerator, with an improvement in receiver sensitivity of $5.6 \mathrm{~dB}$ for a BER of $10^{-4}$.
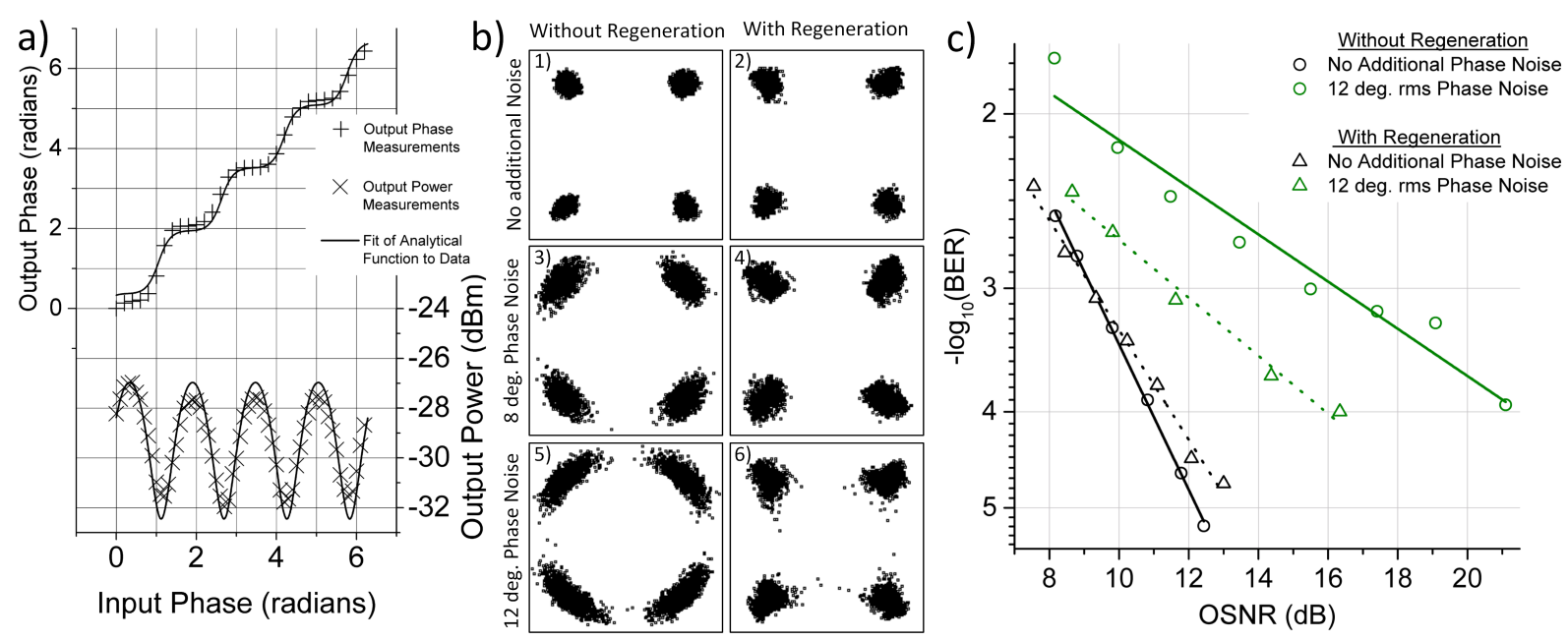

Fig. 3: a) Phase transfer and phase dependent gain profiles, b) Constellation plots for varying phase noise powers, with and without regeneration, c) BERs for signal with and without regeneration.

\section{Conclusion}

We have proposed and demonstrated a wavelength converting idler-free phase quantiser, notable for its low power operation, compactness and flexibility in the choice of operating power. Using the system as a regenerator, BER measurements were taken for a broadband phase noise loaded QPSK signal, illustrating that, for an input signal with phase error equal to $12^{\circ} \mathrm{rms}$, the phase error can be reduced by half and receiver sensitivity can be decreased by $5.6 \mathrm{~dB}$ for a BER of $10^{-4}$.

\section{Acknowledgements}

Research sponsored by EPSRC grant EP/I01196X, The Photonics Hyperhighway. Dr F. Parmigiani is a Royal Academy of Engineering/EPSRC Research Fellow. We thank Dr. R. Slavík for assistance with phase tracking.

\section{References}

1. J. Kakande, R. Slavík, F. Parmigiani, A. Bogris, D. Syvridis, L. Grner-Nielsen, R. Phelan, P. Petropoulos, and D. J. Richardson, "Multilevel quantization of optical phase in a novel coherent parametric mixer architecture," Nature Photonics, vol. 5, no. 12, pp. 748-752, Dec. 2011.

2. M. Asobe, T. Umeki, H. Takenouchi, and Y. Miyamoto, "In-line phase-sensitive amplifier for QPSK signal using multiple QPM LiNbO3 waveguide,” in OECC/PS, 2013 18th, June 2013.

3. F. Parmigiani, R. Slavík, G. Hesketh, P. Petropoulos, and D. Richardson, "Quadrature decomposition of optical fields using two orthogonal phase sensitive amplifiers," in ECOC 2014, Sep. 2014.

4. Z. Zheng, L. An, Z. Li, X. Zhao, J. Yan, and X. Liu, “All-Optical Regeneration of DQPSK/QPSK Signals Based on PhaseSensitive Amplification," in Optical Fiber communication/National Fiber Optic Engineers Conference, 2008. OFC/NFOEC 2008. Conference on, Feb 2008, pp. 1-3.

5. J. Kakande et al., "QPSK phase and amplitude regeneration at 56 Gbaud in a novel idler-free non-degenerate phase sensitive amplifier," in OFC/NFOEC, 2011, 2011.

6. K. Bottrill, G. Hesketh, F. Parmigiani, P. Horak, D. Richardson, and P. Petropoulos, "Suppression of Gain Variation in a PSA-Based Phase Regenerator Using an Additional Harmonic," Photonics Technology Letters, IEEE, vol. 26, no. 20, pp. 2074-2077, Oct 2014. 